\title{
Exit Site Infection in Peritoneal Dialysis; Need for Follow-Up on Cultures Despite Clinical Improvement
}

\author{
Abhilash Koratala Volodymyr Chornyy Amir Kazory \\ Division of Nephrology, Hypertension and Renal Transplantation, University of Florida, Gainesville, FL, USA
}

To the Editor,

Exit site infection is a well-known complication among patients receiving peritoneal dialysis (PD). Common responsible organisms include Staphylococcus aureus and S. epidermidis [1]. Therefore, current guidelines recommend initial empiric antibiotics that are aimed at these organisms. However, infections secondary to uncommon organisms such as atypical mycobacteria and non-diphtheria corynebacteria are being increasingly reported with the widespread use of antimicrobial prophylaxis of the exit site $[2,3]$.

We recently encountered a 59-year-old woman with a history of diabetes mellitus and end-stage renal disease treated with PD who presented with fever, chills, redness, and swelling at her PD catheter exit site for the previous 2 days. She had a failed renal transplant 4 months before and was receiving azathioprine $75 \mathrm{mg}$ /day and prednisone $10 \mathrm{mg} /$ day. Examination revealed erythema and edema at the exit site with serosanguinous discharge. PD fluid cell count was not suggestive of $\mathrm{PD}$-associated peritonitis and gram stain from the exit site was negative. The PD catheter exit site infection was diagnosed, and intravenous antibiotic therapy with vancomycin and cefepime was started, which was followed by rapid clinical improvement. She was discharged home on oral amoxicillin-clavulanic acid. Interestingly, 10 days later, all cultures obtained from the exit site at different time points revealed acid-fast bacilli later identified as Mycobacterium fortuitum. The decision was made to use oral clarithromycin and ciprofloxacin for 2 months. Of note, the patient reported using well water for bathing which could be the potential source for atypical mycobacteria; the culture of the water was performed and was negative for mycobacteria. The patient has remained asymptomatic with normal exit site on examination.

$M$. fortuitum is the most commonly reported non-tuberculous mycobacterium associated with $\mathrm{PD}$-associated peritonitis. However, exit site infection with this organism is very rare [4]. There are 2 key educational points in this case. First, immunosuppressive agents, even at very low dose, could still increase a patient's susceptibility to uncommon and atypical infections. Second, clinical improvement after initial empiric antibiotic therapy in these patients could possibly be due to partial sensitivity of the organisms; it is hence of utmost importance to continue monitoring their culture results despite initial positive response to therapy. Raising awareness of the physicians providing care for these patients could prove helpful in lowering the risk of missed diagnosis and potentially adverse consequences due to lack of appropriate follow-up.

\section{Funding}

None.

\section{Disclosure Statement}

The authors declare that they do not have any conflicts of interest or funding arrangements to disclose relating to this article.

\section{Informed Consent}

Written informed consent was obtained from the patient for publication of this manuscript.

\section{KARGER}

(c) 2017 S. Karger AG, Basel
Abhilash Koratala, MD Division of Nephrology Hypertension and Renal Transplantation PO Box 100224, Gainesville, FL 32610 (USA) E-Mail akoratsla@ufl.edu 


\section{References}

1 Scalamogna A, Castelnovo C, De Vecchi A, Ponticelli C: Exit-site and tunnel infections in continuous ambulatory peritoneal dialysis patients. Am J Kidney Dis 1991;18:674677.
2 Tse KC, Lui SL, Cheng VC, Yip TP, Lo WK A cluster of rapidly growing mycobacterial peritoneal dialysis catheter exit-site infections. Am J Kidney Dis 2007;50:e1-e5.

3 Schiffl H, Mücke C, Lang SM: Exit-site infections by non-diphtheria corynebacteria in CAPD. Perit Dial Int 2004;24:454-459.
4 Gourtzelis N, Margassery S, Bastani B: Successful treatment of severe Mycobacterium fortuitum exit-site infection with preservation of the Tenckhoff catheter. Perit Dial Int 2005;25:607-608. 